\title{
Cobalt Doping in Tantalum Nitride Thin Films Prepared by Sputtering
}

Sam Macartney ${ }^{1}$, Leigh Sheppard ${ }^{2}$ and Richard Wuhrer ${ }^{3}$

${ }^{1}$ Western Sydney University, Sydney, New South Wales, Australia, ${ }^{2}$ Western Sydney University, Penrith, Northern Territory, Australia, ${ }^{3}$ Western Sydney University, Penrith, New South Wales, Australia

The process of solar driven water splitting, employed in theoretical solar hydrogen farms, could provide sustainable hydrogen in a theoretical hydrogen economy [1]. Since the discovery of photocatalytic water splitting using $\mathrm{TiO}_{2}$ [2], four decades of research has been focused on the development of efficient solar to hydrogen water splitting materials. In recent years, various nitride materials have been highlighted as possessing great potential for efficient water splitting [3]. Tantalum nitride, specifically $\mathrm{Ta}_{3} \mathrm{~N}_{5}$, is one such nitride, possessing appropriate band edge positions for efficient, bias free overall water splitting and a band gap allowing absorption of visible light [4].

The goal of this study was to evaluate the impact of cobalt doping on $\mathrm{Ta}_{3} \mathrm{~N}_{5}$, for ultimate use as a photoelectrode. Doping is one of many approaches have been utilised to optimise the properties of $\mathrm{Ta}_{3} \mathrm{~N}_{5}$ for solar water splitting, with the inclusion of metals such as $\mathrm{Zr}$ and $\mathrm{Mg}$ (among others) having been shown to improve the crystallisation of $\mathrm{Ta}_{3} \mathrm{~N}_{5}$, confer band gap control or improve conductivity [5]. Cobalt is a heavily studied dopant species in semiconductors and was selected for this study due to its abundance of electronic states and potential to improve favourable defects in the $\mathrm{Ta}_{3} \mathrm{~N}_{5}$ lattice. Given the relatively immature nature of doped $\mathrm{Ta}_{3} \mathrm{~N}_{5}$ in the literature, it is important to characterise the impact of dopants on the host structure.

Cobalt doped tantalum oxide films were deposited via DC sputtering with the deposition parameters listed in Table 1. A control film was also deposited, with no power on the cobalt target. Films were deposited using an AJA Orion 5 magnetron sputtering system (AJA International, Scituate MA). After deposition, films were annealed in a Labec tube furnace (Labec, Sydney, Australia) at $1173 \mathrm{~K}$ for 12 hours in a 10:90 gaseous mixture of $\mathrm{NH}_{3}: \mathrm{N}_{2}$, flowed at $100 \mathrm{sccm}$. Surface feature imaging and elemental quantification was performed with a ZEISS Merlin FEG (Zeiss, Oberkochen, Germany) Scanning Electron Microscopy (SEM) equipped with a Bruker XFlash $6 \mathrm{I10}$ (Bruker, MA) detector for Energy-Dispersive Spectroscopy (EDS).

The amount of cobalt sputtered had a strong effect on the elemental composition and microstructure of the tantalum nitride films. Surface images of the control and cobalt doped films are displayed in Figure 1. Deposition rate was seen to increase with power on the cobalt target, likely due to the additional presence of cobalt in the films. Cobalt doped films showed smaller grains with significant porosity relative to the Ta3 5 control film, which was also apparent in the cross sections of the doped films. Cobalt presence as indicated by EDS increased with power on the cobalt target, however the presence of cobalt also correlated with the presence of oxygen. It is apparent that cobalt acts through an unknown mechanism to increase the presence of oxygen in the resulting films, even in the film with a very small presence of cobalt $(10 \mathrm{~W}$ : $0.5 \%$ at. \% cobalt). This may necessitate longer annealing times to remove all oxygen from the cobalt doped films. Further kinetic experimentation and phase analysis would be necessary to better understand the precise role cobalt plays. Photoelectrochemical or electronic analysis methods would be necessary to determine the performance properties of the films.

Table 1. Processing parameters for cobalt doped tantalum nitride films, and the resulting film thickness and composition after nitridation. EDS was performed with an accelerating voltage of $10 \mathrm{kV}$. 


\begin{tabular}{|c|c|c|c|c|c|c|c|c|c|}
\hline $\begin{array}{l}\text { Atmospheric } \\
\text { Ar:O2 } \\
(\% \\
\text { atmosphere) }\end{array}$ & $\begin{array}{l}\text { Deposition } \\
\text { Pressure } \\
\text { (mTorr) }\end{array}$ & $\begin{array}{l}\text { Target } \\
\text { Power } \\
\text { (Ta:Co) }\end{array}$ & $\begin{array}{l}\text { Thickness } \\
\text { As } \\
\text { Deposited } \\
(\mathrm{nm})\end{array}$ & $\begin{array}{l}\text { Thickness } \\
\text { Post-NH3 } \\
(\mathrm{nm})\end{array}$ & $\begin{array}{l}\text { Dep. Rate } \\
\text { (nm/min) }\end{array}$ & $\begin{array}{l}{[\mathrm{N}]} \\
(\text { at. \%) }\end{array}$ & $\begin{array}{l}{[\mathrm{Ta}]} \\
(\text { at. \%) }\end{array}$ & $\begin{array}{l}{[\mathrm{O}]} \\
\text { (at. \%) }\end{array}$ & $\begin{array}{l}{[\mathrm{Co}]} \\
(\text { at. \%) }\end{array}$ \\
\hline 90:10 & 5 & $100: 10$ & 607 & 754 & 5.05 & 64.6 & 20.6 & 14.3 & 0.5 \\
\hline 90:10 & 5 & $100: 20$ & 578 & 733 & 4.81 & 56.5 & 22.5 & 17.2 & 3.8 \\
\hline 90:10 & 5 & $100: 30$ & 507 & 853 & 4.22 & 37 & 23.4 & 28.3 & 11.3 \\
\hline $90: 10$ & 5 & 100:0 & 471 & 650 & 3.92 & 71 & 25 & 4 & 0 \\
\hline
\end{tabular}

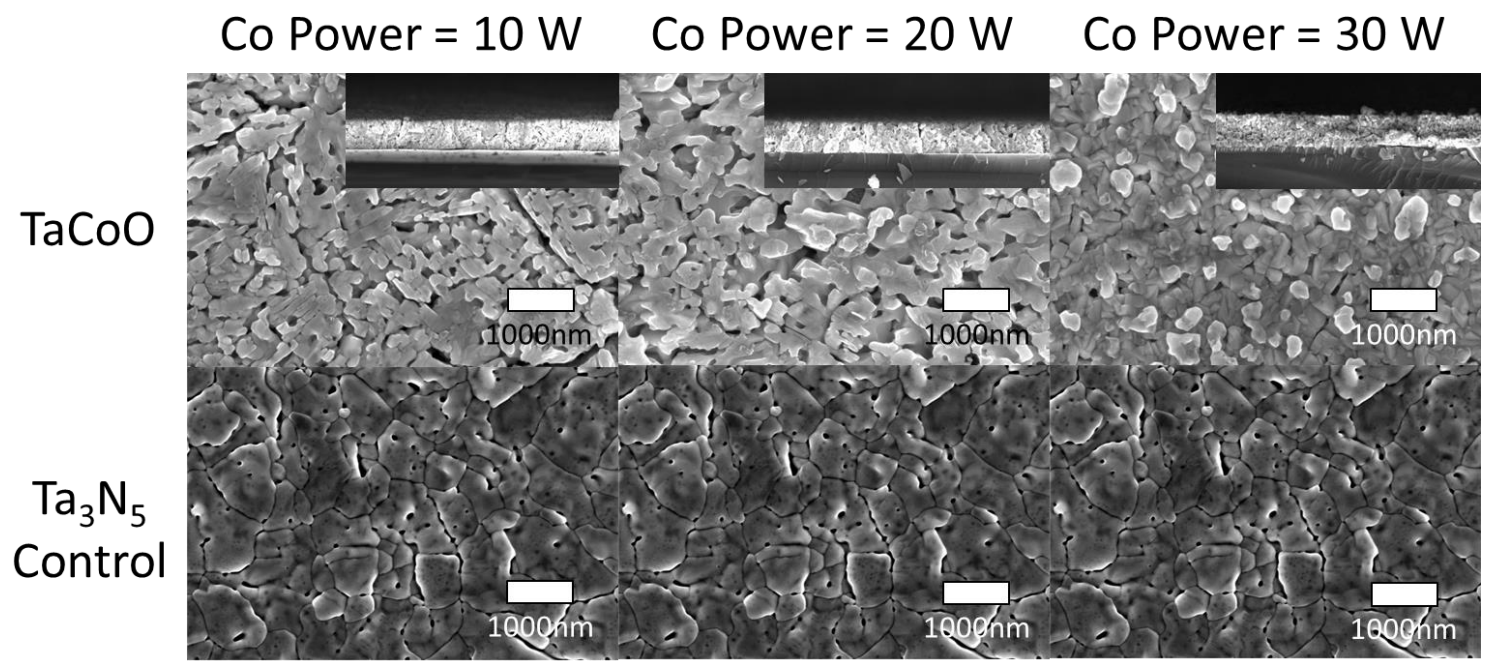

Figure 1. SEM images showing surface features and cross sections of Cobalt doped TaxNy films deposited with 10, 20 and $30 \mathrm{~W}$ on the cobalt target, contrasted with a Ta3N5 film deposited without any cobalt. Surface images were taken at 100,000x, with a working distance of $3.5 \mathrm{~mm}$ and accelerating voltage of $10 \mathrm{kV}$. Cross section images are scaled to reflect the scale bar thickness value accurately.

References

[1] Katsushi, F., K. Takeshi, and O. Kazuhiro, Hydrogen Gas Generation by Splitting Aqueous Water Using n-Type GaN Photoelectrode with Anodic Oxidation. Japanese Journal of Applied Physics, 2005. 44(4L): p. L543.

[2] Fujishima, A. and K. Honda, Photolysis-decomposition of water at the surface of an irradiated semiconductor. Nature, 1972. 238(5385): p. 37-38. 
[3] Maeda, K. and K. Domen, Photocatalytic Water Splitting: Recent Progress and Future Challenges. The Journal of Physical Chemistry Letters, 2010. 1(18): p. 2655-2661.

[4] Hitoki, G., et al., Ta3N5 as a novel visible light-driven photocatalyst $(\lambda<600 \mathrm{~nm})$. Chemistry letters, 2002. 31(7): p. 736-737.

[5] Seo, J., et al., Mg-Zr Cosubstituted Ta3N5 Photoanode for Lower-Onset-Potential Solar-Driven Photoelectrochemical Water Splitting. Journal of the American Chemical Society, 2015. 137(40): p. 12780-12783. 